\title{
EFECTOS DEL CONSUMO DE COCAÍNA SOBRE EL FUNCIONAMIENTO DE LA MEMORIA EPISÓDICA
}

\author{
COCAINE CONSUMPTION EFFECTS ON EPISODIC MEMORY PERFORMANCE \\ Andrea G. Said, Marcela C. López, Josefina Rubiales y Guillermo E. Macbeth
}

\begin{abstract}
El consumo de cocaína se ha relacionado con alteraciones neuropsicológicas que afectan un amplio espectro de funciones cognitivas; sin embargo, el análisis exhaustivo del funcionamiento de la memoria episódica es un área de vacancia y conocer en profundidad sus procesos permitiría planificar intervenciones pertinentes. Objetivo. Analizar el funcionamiento de la memoria episódica en personas con diagnóstico de trastorno por consumo de cocaína. Metodología. Diseño de tipo descriptivo. Muestra ocasional, conformada por 80 personas, pareadas en dos grupos, clínico y control. Para cumplir con el objetivo, se administró el Test de Aprendizaje Verbal España-Complutense. Resultados. El grupo clínico presentó un funcionamiento de la memoria episódica inferior al grupo control, alcanzando menores desempeños en tres indicadores de procesos de codificación (uso espontáneo de estrategias semánticas en recuerdo inmediato $p=.03$ y recuerdo con claves a corto $p=.01$ y largo plazo $p=.01$ ), y dos indicadores de procesos de recuperación (uso de estrategias semánticas en el recuerdo libre a corto plazo $p=.00$ y largo plazo $p=.00$ ). Conclusiones. Estos resultados sugieren que las personas con diagnóstico de trastorno por consumo de cocaína presentan un déficit específico y primario en los procesos de codificación, vinculado con el uso escaso o no sistemático de estrategias semánticas.
\end{abstract}

Palabras clave: codificación, almacenamiento, recuperación, memoria episódica, trastorno por diagnóstico de consumo de cocaína.

Cocaine use has been related to neuropsychological disturbances that allow a wide spectrum of cognitive functions, however, the exhaustive analysis of the functioning of episodic memory is a vacancy area and knowing its in-depth processes would allow planning relevant interventions. Objective: To analyze the functioning of episodic memory in people with a diagnosis of cocaine use disorder. Methodology: Descriptive type design. Occasional sample, made up of 80 people, paired into two groups, clinical and control. To meet the objective The Spain-Complutense Verbal Learning Test was administered. Results: The clinic group presented a lower episodic memory performance than the control group. They achieved lower performances in three indicators of coding processes (spontaneous use of semantic strategies in immediate recall $p=.03$ and recall with short keys $p=.01$ and long-term $p=.01$ ), and two indicators of recovery processes (use of semantic strategies in short-term free memory $p=.00$ and long-term $p=.00$ ). Conclusions: These results suggested that people with a diagnosis of cocaine use disorder present a specific and primary deficit in the coding processes, related to to the little or non-systematic use of semantic strategies.

Keywords: coding, storage, recovery, episodic memory, diagnosis of cocaine use disorder.

Andrea Giselle Said, Facultad de Psicología. Instituto de Psicología Básica, Aplicada y Tecnología (IPSIBAT), Universidad Nacional de Mar del Plata, correspondencia a andrea said@hotmail.com, https://orcid.org/0000-0001-5756-4809.

Marcela Carolina López, Instituto de Psicología Básica, Aplicada y Tecnología (IPSIBAT), Universidad Nacional de Mar del Plata, https://orcid.org/0000-0002-2448-8093.

Josefina Rubiales, Instituto de Psicología Básica, Aplicada y Tecnología (IPSIBAT), Universidad Nacional de Mar del Plata, https://orcid.org/0000-0001-6842-318x.

Guillermo Eduardo Macbeth, Facultad Teresa de Ávila, Centro de Investigación Interdisciplinar en Valores, Integración y Desarrollo Social, Pontificia Universidad Católica Argentina (UCA), https://orcid.org/0000-0001-9843-0524. 
El consumo de sustancias supone un grave problema socio-sanitario a nivel global, que preocupa a los actores del sistema de salud y a las personas que han sufrido consecuencias adversas por esta enfermedad (Fernández-Artamendi \& Weidberg, 2016). Según Bugge et al. (2017) contribuye de forma significativa a la carga mundial de morbilidad, encontrándose en la actualidad entre los primeros veinte factores de riesgo de muerte y discapacidad.

La adicción fue considerada durante mucho tiempo una debilidad moral, asociada a falta de fuerza de voluntad. Actualmente es reconocida como una enfermedad crónica que afecta directamente el funcionamiento cerebral. En las últimas décadas se han realizado importantes avances en el análisis de sus bases neurobiológicas, lo que ha permitido conceptualizarla como un trastorno cerebral crónico que provoca la búsqueda y uso compulsivo de sustancia/s, a pesar de las consecuencias adversas que ésta/s provoque/n (Organización Mundial de la Salud, 2008)

Particularmente el consumo de cocaína constituye un problema de interés mundial, tanto por su elevada tasa de prevalencia, como por las graves consecuencias médicas y sociales que implica (Madoz-Gúrpide, Ochoa-Mangado \& Martínez-Pelegrín, 2009). Estudios realizados en Argentina evidencian que la cocaína, junto con el alcohol, el tabaco, los inhalables, la marihuana y la pasta base son las sustancias que presentan mayor tasa de prevalencia (Abeldaño, Fernández, Ventura \& Estario, 2013). En un relevamiento realizado por la Secretaría de Políticas Integrales sobre Drogas de la Nación Argentina en la ciudad de Mar del Plata (Argentina), la cocaína resultó la sustancia psicoactiva más consumida luego de la marihuana; habiendo sido consumida por un $38,1 \%$ del total de los entrevistados alguna vez en la vida, por un $29 \%$ en el último año y un $12,1 \%$ durante el último mes, constituyendo una de las sustancias principales que han motivado tratamientos en los últimos años, marihuana (27,3\%), cocaína (22,5\%) y alcohol $(22,3 \%)$ (SEDRONAR, 2017a, 2017c).

En este punto es importante considerar que el fenómeno del consumo de sustancias resulta sumamente complejo, pues intervienen variables genéticas, neurobiológicas, cognitivas, comportamentales y ambientales. Actualmente existe consenso acerca de la importancia de realizar investigaciones de abordaje multinivel que permitan interrelacionar aspectos micro y macro en la comprensión de los fenómenos humanos. Si bien el presente trabajo hace foco en el estudio de un proceso cognitivo como la memoria, se analizará considerando las vinculaciones bidireccionales del nivel cognitivo con los niveles restantes, y la influencia del ambiente (Figura 1).

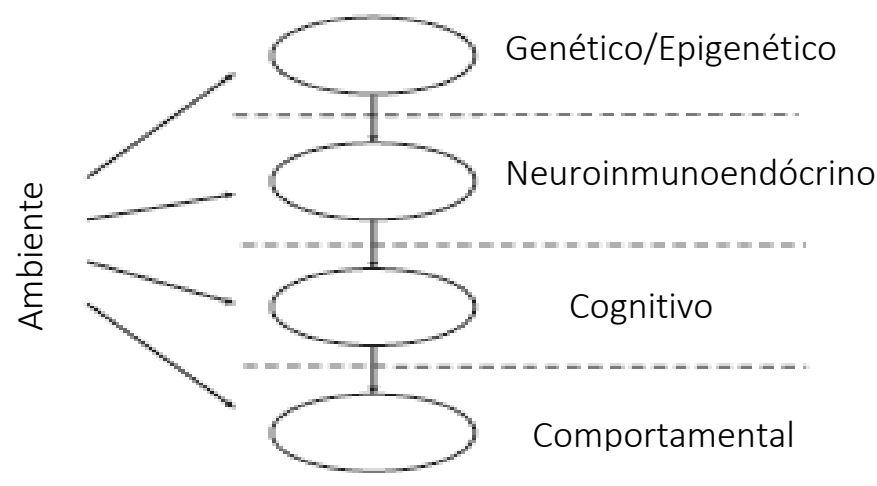

Figura 1. Conceptualización de los diferentes niveles de explicación de las neurociencias cognitivas (adaptado al español de Frith \& Frith, 2010) 
En lo que respecta a los efectos nocivos del consumo de cocaína, son amplios y diversos, afectando el funcionamiento de circuitos cerebrales, debido a daño vascular. Además, provoca modificaciones en receptores y neuromoduladores que tienen importantes consecuencias en el desempeño cognitivo (Madoz-Gúrpide et al., 2009). Dado su mecanismo de acción, resultan especialmente relevantes los daños sobre las vías dopaminérgicas, ampliamente distribuidas en el cerebro y particularmente en el lóbulo frontal (Verdejo-García, Pérez-García, Sánchez-Barrera, Rodríguez-Fernández \& Gómez-Río, 2007). Estudios de neuroimagen revelan alteraciones significativas y estables en los lóbulos frontales y en menor medida en los lóbulos temporales, con descenso de la actividad metabólica, así como una reducción generalizada del flujo sanguíneo cerebral y una disminución específica en la corteza prefrontal, el giro cingulado anterior, el tálamo, los ganglios basales, la corteza occipital y el cerebelo (Montoya-Filardi \& Mazón, 2016).

Asimismo, en relación a procesos cognitivos, las investigaciones reportan alteraciones en atención sostenida, focalizada y alternante; velocidad de procesamiento; habilidades psicomotoras; resolución de problemas; funciones ejecutivas y memoria (Bonet-Álvarez et al., 2015; García-Fernández, García-Rodríguez \& Secades-Villa, 2011; Spronk et al., 2013). En cuanto al funcionamiento de la memoria, si bien los estudios se han abocado al análisis de la memoria de trabajo y la memoria a corto plazo (Madoz-Gúrpide \& Ochoa-Mangado, 2012; Verdejo et al., 2007; Vergara et al., 2010; Vonmoos, Hulka, Preller, Minder, Baumgartner \& Quednow, 2017), demostrando deficiencias particulares en la memoria de trabajo visuoespacial en personas con adicción a la cocaína en comparación con consumidores de otras sustancias (Kübler, Murphy \& Garavan, 2005); Cunha, Nicastri, Gomes, Moino \& Peluso (2004) y Serper et al. (2000) han reportado también dificultades en la memoria de tipo verbal; y Montgomery, Fisk, Newcombe \& Murphy (2005) refieren que en consumidores de éxtasis o de cocaína se encuentran afectados los aspectos semánticos de la memoria. Asimismo, investigaciones actuales refieren dificultades en la memoria episódica fundamentalmente a través de medidas genéricas y en conjunto con otros procesos cognitivos. En este sentido, Fajardo- Balbuca, Carmilema-Tapia \& Sacoto-Molina (2018), utilizando la batería Neuropsi para evaluar los niveles de atención y memoria en consumidores de diferentes sustancias, encontraron que si bien ambas funciones se encuentran alteradas los mayores deterioros se hallan en el área de la memoria, no ligándose estos resultados a una sustancia específica. Por su parte, Vallejo-Reyes (2019) analizaron conjuntamente diferentes funciones neuropsicológicas, entre ellas la memoria episódica declarativa verbal con el Test de Aprendizaje Auditivo Verbal de Rey en un grupo de personas dependientes a la cocaína; encontrando puntajes significativamente menores en la curva de aprendizaje respecto del grupo control. Cabe destacar que los estudios que abordan la memoria episódica resultan escasos, constituyendo el análisis del funcionamiento exhaustivo global de este tipo de memoria y sus procesos un área de vacancia.

La memoria episódica (ME) es considerada como la más elevada de las capacidades mentales, con un desarrollo ontogénico y filogenético tardío y probablemente exclusiva de la especie humana (Tulving, 2002). Tulving (2005) la define como un tipo de recuerdo que se tiene de experiencias pasadas. Constituye un proceso psicológico de primordial importancia para el ser humano, que permite recordar lo que ha pasado mucho tiempo atrás. Sin ella, procesos mentales como el lenguaje o el razonamiento, serían imposibles. Este sistema permite a la persona reexperimentar sucesos, accediendo a una experiencia mental única e inconfundible diferente a conocer o saber algo. Las personas reviven, a través de la conciencia autonoética, experiencias previas y también proyectan experiencias similares al futuro. Esta capacidad supone 
el funcionamiento conjunto de la corteza prefrontal y estructuras del lóbulo medial temporal (hipocampo y amígdala) (Kim \& Yoon, 1998).

Teniendo en cuenta los aspectos dinámicos o procesuales de la memoria, se considera que un adecuado aprendizaje de la información depende del correcto funcionamiento de una sucesión de operaciones diferenciadas e identificadas como la codificación, el almacenamiento y la recuperación. Así, la memoria puede definirse como un proceso psicológico que se ocupa de codificar, almacenar y recuperar la información (Ballesteros-Jiménez, 2012).

De acuerdo a lo expuesto, y considerando la relevancia de la memoria en el aprendizaje y la ausencia de evidencias científicas sobre las características específicas de la ME en personas con diagnóstico de trastorno por consumo de cocaína, la presente investigación pretende aportar evidencias empíricas sobre el funcionamiento de la ME en este población a través de un modelo multinivel, considerando que las personas con diagnóstico por consumo de cocaína presentan alteraciones en circuitos cerebrales específicos que involucran áreas corticales y subcorticales críticas para un adecuado funcionamiento de los procesos de la ME.

Cabe destacar que, desde una perspectiva dinámica o procesual, pueden presentarse problemas de memoria de diferente naturaleza que afecten ya sea a uno o a varios procesos de memoria en forma conjunta. La posibilidad de identificar cuál o cuáles de estos procesos se encuentran afectados y cómo esa afectación genera efectos sobre el funcionamiento de los restantes es importante para la práctica educacional y clínica, en este sentido el trabajo pretende determinar si existe un perfil diferencial en el funcionamiento de los procesos de ME asociado al consumo de cocaína.

\section{Método}

\section{Diseño}

Se utilizó un diseño de tipo descriptivo con dos grupos, de acuerdo a la clasificación de Hernández-Sampieri, Fernández-Collado \& Baptista-Lucio (2014). Se evaluaron variables de funcionamiento global de la ME y de los procesos de codificación, almacenamiento y recuperación.

\section{Participantes}

Muestra ocasional, conformada por 80 personas del partido de General Pueyrredón, dividida en dos grupos.

1) Grupo clínico $(n=40)$, conformado por personas con edades comprendidas entre 18 y 54 años ( $M=30,26 \mathrm{DS}=9,74 ; 20$ personas entre 18 y 29 años, 12 personas entre 30 y 41 años y 8 personas entre 42 y 54 años), con diagnóstico médico de trastorno por consumo de cocaína, siendo ésta la sustancia de consumo principal (considerando que el monoconsumo resulta difícil de encontrar en la clínica siendo el policonsumo el patrón de consumo más frecuente), que realizan tratamiento terapéutico. La media de tiempo de consumo en años fue de 9.81 años (DS=6,32). La media de tiempo de tratamiento en meses fue 4,26 ( $D S=4,34)$. En cuanto al nivel educativo un 68,4\% del grupo no había concluido sus estudios secundarios o tenían un nivel educativo menor, un 18,4\% había 
finalizado el secundario y un $13,2 \%$ tenían estudios terciarios y/o universitarios completos o incompletos.

Los criterios de inclusión para la muestra clínica fueron: tener entre 18 y 60 años, contar con diagnóstico médico de trastorno por consumo de cocaína, siendo ésta la única sustancia consumida o la sustancia principal y estar en tratamiento terapéutico de internación o ambulatorio. Confirmación del diagnóstico de trastorno por consumo de cocaína a partir del cuestionario adaptado a los criterios del Manual Diagnóstico y Estadístico de los trastornos mentales, quinta edición, DSM-5 (American Psychiatric Association, 2013).

2) Grupo control $(n=40)$, conformado por los respectivos controles pareados de forma intencional con el grupo clínico, por sexo, edad y nivel educativo de modo que ambos grupos puedan ser comparables. De igual modo que el grupo clínico, el grupo control estuvo compuesto por personas con edades comprendidas entre 18 y 54 años ( $M=29,64$, $D S=10,63$ ) y se consideró que sea equitativo también el nivel educativo (un 63,9\% del grupo tenía secundario incompleto o un nivel educativo menor, un $22,2 \%$ secundario completo y un $13,9 \%$ estudios terciarios y/o universitarios completos o incompletos).

Los criterios de inclusión de la muestra control fueron: tener entre 18 y 60 años, no tener afecciones psiquiátricas y/o neurológicas, no cumplir los criterios para diagnóstico de trastornos por consumo de cocaína ni otras sustancias psicoactivas de acuerdo a DSM-5 y no estar ni haber estado bajo tratamiento terapéutico ligado al consumo de sustancias psicoactivas. Para descartar afecciones psiquiátricas y/o neurológicas o diagnóstico de trastorno por consumo de sustancias se administró un cuestionario basado en los criterios del DSM-5.

\section{Instrumentos}

Para evaluar el funcionamiento de la ME y los procesos de codificación, almacenamiento y recuperación se utilizó el Test de Aprendizaje Verbal España-Complutense (TAVEC), de Benedet \& Alejandre (1998), adaptación española del California Verbal Learning Test - CVLT (Delis, Kramer, Kaplan \& Ober, 1987). EI TAVEC presenta una fiabilidad estimada (el test completo) de 0.81 (error típico: 4.98). Dicha prueba permite evaluar la curva y estabilidad del aprendizaje, la retención de la información inmediata, a corto y a largo plazo, el uso de estrategias de aprendizaje, la recuperación y el reconocimiento, además de la susceptibilidad a la interferencia.

Se utilizó el procedimiento estandarizado por el manual del instrumento. EI TAVEC consta de una lista de aprendizaje (lista A), una de interferencia (lista B) y una de reconocimiento. Se aplicaron cinco ensayos de aprendizaje auditivo verbal de las palabras de la lista $\mathrm{A}$. Tras el quinto ensayo de la lista A, se leyó la lista B y la persona debió recordar los elementos que la componen. Inmediatamente después, se realizó una prueba de recuerdo libre a corto plazo de la lista $A$, seguida de una prueba de recuerdo con claves semánticas a corto plazo, en la que se mencionó una a una las cuatro categorías semánticas que agrupan a las palabras y se solicitó el recuerdo de las palabras de la lista $\mathrm{A}$ incluidos en cada una de ellas. Pasados 20 minutos se aplicó la prueba de recuerdo libre a largo plazo de la lista $\mathrm{A}$ y, posteriormente, la prueba de recuerdo con claves semánticas a largo plazo. Finalmente, se administró la prueba de reconocimiento, en la que se debe reconocer a las palabras de la lista A entre distractores de la lista B y análogos fonológicos y semánticos. El instrumento permitió obtener una medida del nivel de funcionamiento global de 
la ME global o aprendizaje y un conjunto de índices discriminados que fueron utilizados en este estudio para la evaluación de los procesos de codificación, almacenamiento y recuperación (Tabla 1).

Tabla 1

Índices del TAVEC

\begin{tabular}{l|l}
\hline & Aprendizaje lista A ensayo 1 (Ri-A1) \\
Indices de funcionamiento & Aprendizaje lista A ensayo 5 (Ri-AS) \\
global de la memoria & Aprendizaje total lista A (Ri-AT) \\
& Recuerdo libre corto plazo (RL-CP) \\
& Recuerdo libre largo plazo (RL-LP) \\
& Aciertos en la lista de reconocimiento (AC) \\
\hline Índices de funcionamiento & Perseveraciones (P) \\
atencional de la memoria ${ }^{1}$ & Intrusiones en recuerdo libre (IRL) \\
& Intrusiones en recuerdo con claves (IRCI) \\
& Falsos positivos (FP) \\
\hline \multirow{5}{*}{ Indices de codificación } & Estrategias semánticas lista A (Esem Ri-A) \\
& Estrategias seriales lista A (Eser Ri-A) \\
& Recuerdo con claves corto plazo (RCL-CP) \\
& Recuerdo con claves largo plazo (RCL-LP) \\
& Discriminabilidad \\
\hline Índice de almacenamiento & RL-LP - RL-CP2 \\
\hline \multirow{2}{*}{ Indices de recuperación } & Aciertos en la lista de reconocimiento (AC) \\
& Sesgo \\
& Recuerdo libre corto plazo (RL-CP) \\
& Recuerdo libre largo plazo (RL-LP) \\
& Estrategias semánticas recuerdo libre corto plazo (Esem RL-CP) \\
& Estrategias semánticas recuerdo libre largo plazo (Esem RL-LP) \\
& Estrategias seriales recuerdo libre largo plazo (Eser RL-CP) \\
& Estrategias seriales recuerdo libre largo plazo (Eser RL-LP) \\
& Aciertos en la lista de reconocimiento frente a recuerdo libre a largo \\
plazo (AC/RL-LP) & Aciertos en la lista de reconocimiento frente a recuerdo con claves a \\
& largo plazo (AC/RCI-LP) \\
\hline
\end{tabular}

\section{Procedimiento}

Para la conformación del grupo clínico se accedió a la población a partir de convenios marco preestablecidos por la Facultad de Psicología de la Universidad Nacional de Mar del Plata (UNMDP) con centros de atención de adicciones. Y para la conformación del grupo control se

${ }^{1}$ Ligadas al control ejecutivo y monitoreo de la información, para poder situar un evento en su contexto organizacional o histórico (Benedet \& Alejandre, 1998).

${ }^{2}$ Comparación del rendimiento en las pruebas de RLCP y RLLP, para inferir hasta cierto punto (ya que entre ellas se administra la prueba de recuerdo con claves) los efectos del paso del tiempo. Fórmula: (RLCP-RLLP)/RLLP) ${ }^{\star 100}$. La pérdida significativa de palabras en RLLP respecto a la prueba de RLCP, se interpreta como un defecto en la retención de la información previamente adquirida. 
accedió a la población a partir de convenios marco preestablecidos por la Facultad de Psicología de la (UNMDP con instituciones educativas y laborales de la comunidad. A través de los directivos de dichas instituciones se estableció contacto con las personas dispuestas a participar en la investigación.

\section{Análisis de datos}

Se realizaron estadísticos descriptivos y prueba no paramétrica $U$ de Mann Whitney para dos muestras independientes, ya que no se cumplen los supuestos de normalidad y homocedasticidad de acuerdo al Test de Levene (Pardo \& Ruiz, 2002). Además, en aquellos indicadores en los que obtuvieron diferencias significativas $(p \leq .05)$ se calculó la magnitud del efecto, a través de la Delta de Cliff, donde +1.0 o -1.0 indica la ausencia de superposición entre los dos grupos, mientras que 0.0 indica que las distribuciones de grupos se superponen completamente. Cuando se obtiene un valor de $p$ significativo, se espera que el tamaño del efecto asociado esté cerca de +1.0 o-1.0 debido a que la diferencia entre los grupos es relevante. Cabe destacar que el signo de la Delta de Cliff es irrelevante, importando su valor absoluto, cuando se aleja de cero como indicador de la magnitud del efecto (Macbeth, Razumiejczyk \& Ledesma, 2011). Un valor de .147 se considera un tamaño del efecto pequeño, .33 mediano y .471 grandes (Romano, Kromrey, Coraggio, Skowronek \& Devine, 2006).

\section{Consideraciones éticas}

La participación fue voluntaria y sujeta a consentimiento informado. Se respetaron los principios éticos para la investigación con seres humanos ${ }^{3}$, y las regulaciones argentinas e internacionales que protegen los procesos de investigación. Se procuraron las condiciones necesarias para proteger la confidencialidad de la identidad y datos de los participantes; de acuerdo a la Ley 25.326 de Protección de Datos Personales ${ }^{4}$. Los procedimientos implementados fueron aprobados por el Comité de Ética del Programa Temático Interdisciplinario en Bioética, dependiente de la Secretaría de Ciencia y Técnica de la UNMDP 5 .

\section{Resultados}

Los resultados se presentan considerando en primer lugar el funcionamiento de la ME y luego un análisis detallado de los procesos implicados (codificación, almacenamiento y recuperación).

En la Tabla 2 se muestran los estadísticos descriptivos de los indicadores del TAVEC que valoran el funcionamiento global de la ME, así como los resultados del análisis no paramétrico para dos muestras independientes y la magnitud del efecto.

\footnotetext{
${ }^{3}$ Estipulados por la Declaración de Helsinki y la Ley 11044 del Ministerio de Salud de la Provincia de Buenos Aires, artículo 5 y su decreto reglamentario.

${ }^{4}$ La Dirección Nacional de Datos Personales (Órgano de control de la ley 25.326,) tiene la atribución de atender las denuncias y reclamos que se interpongan con relación al incumplimiento de las normas sobre protección de datos personales.
}

${ }^{5}$ Magister Susana La Rocca, Coordinadora del Programa Temático Interdisciplinario en Bioética (PTIB) de la UNMDP. 
Tabla 2

Estadísticos descriptivos e inferenciales de los índices correspondientes al funcionamiento global de la ME para el grupo clínico y el grupo control

\begin{tabular}{lcccccc}
\hline & $\begin{array}{c}\text { Clínico } \\
(n=40)\end{array}$ & $\begin{array}{c}\text { Control } \\
(n=40)\end{array}$ & $U$ & $Z$ & $p$ & $D$ \\
& $\mathrm{M}(\mathrm{DS})$ & $\mathrm{M}(\mathrm{DS})$ & & & & \\
\hline Ri-A1 & $6.97(1.73)$ & $6.72(1.63)$ & 635.00 & -.72 & .47 & $\rightarrow 0$ \\
Ri- A5 & $11.64(2.87)$ & $1236(1.93)$ & 604.50 & $-1,04$ & .30 & -.13 \\
Ri-AT & $49.44(10.67)$ & $51.00(7.96)$ & 665.00 &,- 39 & .69 & $\rightarrow 0$ \\
RL-CP & $10.59(2.29)$ & $10.94(2.51)$ & 653.00 & -.52 & .60 & $\rightarrow 0$ \\
RL-LP & $11.54(2.24)$ & $11.78(2.01)$ & 650.00 & -.55 & .58 & $\rightarrow 0$ \\
AC & $15.15(1.28)$ & $15.39(0.72)$ & 686.00 & -.85 & .85 & $\rightarrow 0$ \\
\hline
\end{tabular}

Nota: U=U Mann-Whitney, D=Delta de Cliff, Ri-A1=Aprendizaje lista A ensayo 1, Ri-A5=Aprendizaje lista A ensayo 5, Ri-AT=Aprendizaje total lista A, RL-CP=Recuerdo libre corto plazo, RL-LP=Recuerdo libre largo plazo, $\mathrm{AC}=$ Aciertos en la lista de reconocimiento.

Las deltas $\rightarrow 0$ son coherentes con la no significación estadística hallada.

En la Tabla 2 se observa que el desempeño de las personas con diagnóstico de trastorno por consumo de cocaína en los indicadores que valoran el funcionamiento global de la ME, tiende a ser inferior que el desempeño del grupo control. Como no se detectaron diferencias significativas mediante comparaciones generales, se realizaron comparaciones específicas de los aspectos más relevantes del funcionamiento de la memoria. Se estudiaron el número de perseveraciones e intrusiones en la totalidad de las pruebas y los falsos positivos en la prueba de reconocimiento, planteados en la Tabla 1 como indicadores del funcionamiento atencional de la memoria y de la calidad del recuerdo.

Tabla 3

Estadísticos descriptivos e inferenciales de los índices correspondientes al funcionamiento atencional de la memoria para el grupo clínico y el grupo control

\begin{tabular}{lcccccc}
\hline & $\begin{array}{l}\text { Clínico } \\
(n=40)\end{array}$ & $\begin{array}{c}\text { Control } \\
(n=40)\end{array}$ & $U$ & $Z$ & $p$ & $D$ \\
& $\mathrm{M}(\mathrm{DS})$ & $\mathrm{M}(\mathrm{DS})$ & & & & \\
& $8.28(7.17)$ & $7.25(5.19)$ & 654.50 & -.50 & .61 & $\rightarrow 0$ \\
$\mathrm{P}$ & $3.56(3.47)$ & $2.22(2.45)$ & $526.50^{*}$ & -1.89 & .05 & .25 \\
$\mathrm{IRL}$ & $2.00(2.57)$ & $.83(1.42)$ & $463.00^{* *}$ & -2.68 & .00 & .34 \\
$\mathrm{IRCl}$ & $1.00(1.48)$ & $.47(.84)$ & 561.50 & -1.69 & .09 & .20 \\
$\mathrm{FP}$ & & & & & & \\
\hline
\end{tabular}

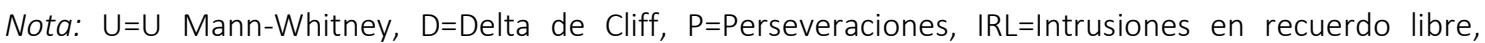
IRCl=Intrusiones en recuerdo con claves, $\mathrm{FP}=$ Falsos positivos.

${ }^{* *} \mathrm{p}<.01-{ }^{*} \mathrm{p}<.05$

En la Tabla 3, se presentan los estadísticos descriptivos de dichos indicadores, así como los resultados del análisis de la prueba U Mann-Whitney para dos muestras independientes y la magnitud del efecto.

Las personas con diagnóstico de trastorno por consumo de cocaína obtuvieron puntuaciones superiores en los cuatro indicadores analizados, presentando diferencias estadísticamente 
significativas en intrusiones en recuerdo libre $(p=.05)$ y en intrusiones en recuerdo con claves $(p=.00)$, con una magnitud del efecto positiva pequeña y mediana, respectivamente, que refleja que el grupo clínico presenta mayores valores que el grupo control, y por ende, al tratarse de errores en la calidad del recuerdo, menores rendimientos.

A continuación, se presentan los resultados detallados de los procesos de codificación, almacenamiento y recuperación.

En la Tabla 4 se presentan las puntuaciones obtenidas en los indicadores que valoran el proceso de codificación.

Tabla 4

Estadísticos descriptivos e inferenciales de los índices correspondientes a los indicadores del proceso de codificación

\begin{tabular}{lcccccc}
\hline & $\begin{array}{c}\text { Clínico } \\
(n=40)\end{array}$ & $\begin{array}{c}\text { Control } \\
(n=40)\end{array}$ & $U$ & $Z$ & $p$ & $D$ \\
& $\mathrm{M}(\mathrm{DS})$ & $\mathrm{M}(\mathrm{DS})$ & & & & \\
\hline Esem Ri-A & $12.26(7.47)$ & $15.72(7.95)$ & $502.00^{*}$ & -2.12 & .03 & -.28 \\
Eser Ri-A & $4.95(3.30)$ & $5.08(3.40)$ & 688.50 & -.14 & .89 & $\rightarrow 0$ \\
RCL-CP & $11.49(2.19)$ & $12.72(1.95)$ & $466.50^{*}$ & -2.52 & .01 & -.33 \\
RCL-LP & $11.72(2.31)$ & $13.06(1.85)$ & $460.50^{*}$ & -2.58 & .01 & -.34 \\
Discriminabilidad & $95.79(3.96)$ & $97.56(2.76)$ & $515.50^{*}$ & -2.03 & .04 & -.25 \\
\hline
\end{tabular}

Nota: U=U Mann-Whitney, D=Delta de Cliff, Esem Ri-A=Estrategias semánticas lista A, Eser Ri-A=Estrategias seriales lista $\mathrm{A}, \mathrm{RCL}-\mathrm{CP}=$ Recuerdo con claves corto plazo, RCL-LP=Recuerdo con claves largo plazo.

${ }^{*} p<.05$

Los resultados indican diferencias estadísticamente significativas en cuatro de los indicadores analizados, obteniendo las personas con diagnóstico de trastorno por consumo de cocaína desempeños inferiores, con magnitudes del efecto negativas pequeñas y medianas. Estos datos evidencian un desempeño menor del grupo clínico en relación al grupo control, tanto cuando se compara la utilización espontánea de estrategias semánticas en el recuerdo inmediato $(p=.03)$ así como en las pruebas de recuerdo con claves a corto $(p=.01)$ y largo plazo $(p=.01)$ que implican la posibilidad de valerse de claves proporcionadas por el evaluador para organizar la información. El único índice que no presentó diferencias significativas corresponde al uso de estrategias seriales, que no constituye un indicador crítico en un proceso de codificación exitosa.

En relación al proceso de almacenamiento, para evaluar la información retenida a pesar del paso del tiempo, se utilizó el índice propuesto por el TAVEC: recuerdo libre a largo plazo frente a recuerdo libre a corto plazo, no encontrándose diferencias significativas entre ambos grupos (Tabla 5). 
Tabla 5

Estadísticos descriptivos e inferenciales del índice de desempeño en tareas de almacenamiento en el grupo clínico y control

\begin{tabular}{ccccccc}
\hline & Clínico & Control & U & Z & $p$ & $D$ \\
& $(n=40)$ & $(n=40)$ & & & & \\
M (DS) & M (DS) & & & & \\
\hline RL-LP - RL-CP & $-7.62(13.78)$ & $-6.91(15.06)$ & 632.50 & -.74 & .46 & $\rightarrow 0$ \\
\hline
\end{tabular}

Nota: $\mathrm{U}=\mathrm{U}$ Mann-Whitney, $\mathrm{D}=$ Delta de Cliff, $\mathrm{RL}-\mathrm{LP}=$ Recuerdo libre largo plazo, $\mathrm{RL}-\mathrm{CP}=$ Recuerdo libre corto plazo.

La delta $\rightarrow 0$ son coherentes con la no significación estadística hallada.

Por último, completando el análisis procesual, en la Tabla 6 se analizan los indicadores que valoran el proceso de recuperación.

En este caso, y en consonancia con lo analizado en el proceso de codificación, se observan diferencias significativas en el uso de estrategias semánticas en las pruebas de recuerdo a corto plazo $(p=.00)$, y largo plazo $(p=.00)$, presentando las personas con diagnóstico de trastorno por consumo de cocaína valores inferiores. Se observan magnitudes del efecto pequeñas y medianas, de signo negativo, evidenciando un desempeño menor del grupo clínico en relación al grupo control en la utilización de estrategias semánticas en las pruebas de recuerdo a corto y largo plazo.

Tabla 6

Estadísticos descriptivos e inferenciales de los índices correspondientes a los procesos de recuperación en el grupo clínico y control

\begin{tabular}{lcccccc}
\hline & $\begin{array}{c}\text { Clínico } \\
(n=40)\end{array}$ & $\begin{array}{c}\text { Control } \\
(n=40)\end{array}$ & $U$ & $Z$ & $p$ & $D$ \\
& $\mathrm{M}(\mathrm{DS})$ & $\mathrm{M}(\mathrm{DS})$ & & & & \\
\hline AC & $15.15(1.28)$ & $15.39(.72)$ & 686.00 & -.85 & .85 & $\rightarrow 0$ \\
Sesgo & $.00(0.31)$ & $.00(.17)$ & 693.50 & -.11 & .91 & $\rightarrow 0$ \\
RL-CP & $10.59(2.29)$ & $10.94(2.51)$ & 653.00 & -.52 & .60 & -.04 \\
RL-LP & $11.54(2.24)$ & $11.78(2.01)$ & 650.00 & -.55 & .58 & $\rightarrow 0$ \\
Esem RL-CP & $3.54(2.48)$ & $5.00(2.42)$ & $455.50^{* *}$ & -2.63 & .00 & -.35 \\
Esem RL-LP & $4.36(2.57)$ & $6.11(2.35)$ & $435.50^{* *}$ & -2.84 & .00 & -.37 \\
Eser RL-CP & $.51(0.79)$ & $.39(.72)$ & 651.00 & -.66 & .51 & $\rightarrow 0$ \\
Eser RL-LP & $.64(0.87)$ & $.31(.52)$ & 576.50 & -1.58 & .11 & .17 \\
AC/RL-LP & $-23.65(14.74)$ & $-23.75(11.33)$ & 701.00 & -.01 & .99 & $\rightarrow 0$ \\
AC/RCl-LP & $-22.22(16.07)$ & $-15.27(10.44)$ & $501.00 *$ & -2.13 & .03 & -.29 \\
\hline
\end{tabular}

Nota: $\mathrm{U}=\mathrm{U}$ Mann-Whitney, $\mathrm{D}=$ Delta de Cliff, $\mathrm{AC}=$ Aciertos en la lista de reconocimiento, $\mathrm{RL}-\mathrm{CP}=$ Recuerdo libre corto plazo, RL-LP=Recuerdo libre largo plazo, Esem RL-CP=Estrategias semánticas recuerdo libre corto plazo, Esem RL-LP=Estrategias semánticas recuerdo libre a largo plazo, Eser $\mathrm{RL}-\mathrm{CP}=$ Estrategias seriales recuerdo libre a corto plazo, Eser RL-LP=Estrategias seriales recuerdo libre a largo plazo, $A C / R L-$ $\mathrm{LP}=$ Aciertos en la lista de reconocimiento frente a recuerdo libre a largo plazo, $\mathrm{AC} / \mathrm{RCl}-\mathrm{LP}=\mathrm{Aciertos}$ en la lista de reconocimiento frente a recuerdo con claves a largo plazo.

${ }^{* *} p<.01-{ }^{*} p<.05$ 
Por otra parte, si bien ambos grupos presentan un desempeño similar en la prueba de reconocimiento (recuperación pasiva), uno de los índices analizados $(A C / R C l-L P)$ que refleja memoria estratégica (recuperación activa) presenta diferencias significativas ( $p=.03$ ) con un mejor desempeño del grupo control.

\section{Discusión}

La literatura científica reporta que el consumo de cocaína se ha relacionado con alteraciones neuropsicológicas en un amplio espectro de funciones cognitivas, vinculadas a la desregulación funcional de diversas regiones cerebrales (Bonet-Álvarez et al., 2015; Spronk et al., 2013). Sin embargo, los estudios actuales sobre ME son escasos, siendo evaluada en forma conjunta con otros procesos cognitivos a través de medidas genéricas, por lo que la presente investigación se propuso analizar exhaustivamente el funcionamiento global de la ME en personas con diagnóstico de trastorno por consumo de cocaína, a través de un modelo de análisis multinivel.

En primer lugar, en relación a los índices generales de funcionamiento global de la ME, la población clínica ha presentado desempeños inferiores, con diferencias en la calidad del recuerdo, reflejado en un mayor número de errores. Estos resultados pueden considerarse como alteraciones de las funciones del procesador central, planteadas por los autores del instrumento utilizado (Benedet \& Alejandre, 1998). Este sistema se encarga de situar un evento en su contexto organizacional e histórico y posee, en su operatoria, funciones atencionales o ejecutivas y funciones de procesamiento estratégico u organizativas. Las primeras, refieren a la capacidad de chequeo y monitoreo de las propias conductas y al feedback realizado a partir de las respuestas del entorno y pueden evaluarse a partir de la capacidad para mantener el uso de una estrategia de aprendizaje una vez que ha sido descubierta y de la presencia de perseveraciones ${ }^{6}$ e intrusiones?.

Asimismo, de modo general, estos resultados podrían asociarse a las dificultades en las funciones ejecutivas reportadas en personas con diagnóstico de trastorno por consumo de cocaína (González-Roscigno, Mujica-Díaz, Terán-Mendoza, Guerrero-Alcedo \& Arroyo-Alvarado, 2015). Dichas funciones tienen como sustrato neuroanátomico fundamental a los lóbulos prefrontales y son esenciales para un adecuado funcionamiento social diario. Permiten llevar a cabo una conducta eficaz, creativa y socialmente aceptada, involucrando el establecimiento de metas, la planificación y programación, la supervisión de las tareas y el feedback, la ejecución efectiva, la inhibición de conductas distractoras, el juicio y el razonamiento abstracto (MadozGúrpide \& Ochoa-Mangado, 2012). Al mismo tiempo, estos resultados son particularmente consistentes con las fallas en el control inhibitorio asociadas a esta población, que se caracterizan por conductas impulsivas que brindan satisfacción inmediata a expensas de otras conductas que pueden resultar más ventajosas a medio o largo plazo (Romero-Ayuso, Mayoral-Gontán \& TriviñoJuárez, 2016). Así, la población clínica evidencia dificultades para dejar de lado la interferencia

\footnotetext{
${ }^{6}$ Pueden ser de dos tipos: El primero indica fallas del sistema supervisor prefrontal y falta de control mental sobre las palabras que se van produciendo, generalmente acompañado de expresiones como no sé si lo he dicho ya. El otro tipo, puede aparecer desde el comienzo del recitado de la lista y consiste en que una respuesta previa aparece inadecuadamente de modo repetido (perseveraciones recurrentes) interfiriendo en el procesamiento normal de la información.

${ }^{7}$ Implican dificultades en la discriminación que el recuerdo o el reconocimiento de una lista de palabras supone. Pueden presentarse en las pruebas de recuerdo libre (inmediato, a corto y largo plazo), en las pruebas de recuerdo con clave (a corto y largo plazo) o en la prueba de reconocimiento (falsos positivos). que son aquellas intrusiones que tienen lugar en las pruebas de reconocimiento.
} 
que suponen las palabras de la otra lista presentada o el propio sistema semántico preexistente, así como para inhibir el impulso de repetir una palabra ya dicha.

Cabe destacar que, desde el modelo multinivel, es importante considerar las relaciones entre los circuitos cerebrales anatómica y funcionalmente alterados, los problemas neuropsicológicos que estos traen aparejados, así como su influencia en el comportamiento de la persona. MadozGúrpide \& Ochoa-Mangado (2012) señalan relaciones entre determinadas disfunciones cognitivas y el mantenimiento de la conducta adictiva, especialmente en lo que respecta a la tendencia a la perseveración, la incapacidad para la inhibición de impulsos irrelevantes, las dificultades para procesar y aprender de los errores, y la impulsividad. La desregulación funcional de los lóbulos frontales, y las alteraciones cognitivas asociadas, impactan negativamente en la vida diaria, en el funcionamiento ocupacional y social de la persona (Mendoza-Carmona, LópezGuerra y Cuello-Prato, 2019). Asimismo, juegan un papel esencial en la perpetuación del consumo problemático, condicionando el pronóstico evolutivo y los resultados terapeúticos, por lo que deben ser considerados en el desarrollo de programas de prevención y tratamiento (MadozGúrpide et al., 2009).

En relación al análisis procesual de la ME, al examinar el proceso de codificación se observan rendimientos menores de las personas con diagnóstico de trastorno por consumo de cocaína en el uso de estrategias semánticas y en los índices recuerdo con claves corto plazo, recuerdo con claves largo plazo y discriminabilidad. De acuerdo a lo planteado por López y Muñoz (2012), el uso de estrategias de memoria potencia el aprendizaje y posterior recuerdo. A su vez, constituye la principal medida de valoración del proceso de codificación (Gershberg \& Shimamura, 1995) ya que implica un procesamiento complejo de la información, que supone la agrupación por categorías o el establecimiento de relaciones semánticas entre los estímulos (Introzzi, Urquijo, Richard's, Canet-Juric \& Richaud, 2012). Supone un esfuerzo y control cognitivo para ordenar una situación nueva, donde las respuestas automáticas resultan insuficientes (Collette, Hogge, Salmon \& Van der Linden, 2006). Existe acuerdo que, para el aprendizaje de listas de cierta extensión como la empleada en esta investigación, del conjunto de estrategias mnemotécnicas, el agrupamiento y la categorización parecen ser más eficaces que otros recursos como la simple repetición (Sohlberg \& Mateer, 2001) o el uso de estrategias seriales (Benedet, Martínez-Arias \& Alejandre, 1998). En este sentido, las personas con diagnóstico de trastorno por consumo de cocaína implementaron un número menor de estrategias semánticas, y al mismo tiempo, un mayor uso de estrategias seriales; resultado que puede interpretarse como evidencia a favor de dificultades en el proceso de codificación.

Por otra parte, diferentes investigaciones consideran que el desempeño en las pruebas de recuerdo con claves permite hacer ciertas inferencias acerca de si el problema de memoria puede ser explicado por dificultades en el proceso de codificación o en los procesos de recuperación (Delis et al., 1987, citado en Benedet \& Alejandre, 1998). De los resultados presentados se desprende que la población clínica no organizó la información de acuerdo a categorías semánticas durante el proceso de codificación ya que, si así hubiese sido, la ayuda o clave semántica proporcionada externamente en un momento posterior hubiera permitido un desempeño superior con respecto a las pruebas de recuerdo libre.

Por último, los rendimientos menores en el índice de discriminabilidad señalan que la población clínica presenta dificultades en los mecanismos de análisis inter-ítem e intra-ítem que posibilitan extraer información relacional y distintiva del material de aprendizaje, y por ende, un 
aprendizaje discriminado (Einstein \& Hunt, 1980). El mayor número de falsos positivos refleja dificultades en la discriminación de las palabras de la lista presentada con otras que tienen semejanza fonológica, semántica o que integraban otra lista. Siguiendo a Benedet \& Alejandre (1998), aprender no significa simplemente almacenar información, sino almacenarla discriminadamente para posibilitar una codificación exitosa.

Teniendo en cuenta los resultados encontrados, podría considerarse que las personas con diagnóstico de trastorno por consumo de cocaína tendrían dificultades para el establecimiento de una estrategia sistemática y eficaz durante la codificación de la información, como pueden ser las estrategias semánticas. No lograrían desarrollar un criterio organizacional y sostenerlo en el tiempo, haciendo uso de una codificación más automática y repetitiva. Esto podría ligarse a las características compulsivas del consumo, que se sostiene en forma impulsiva y sin control, incluso a pesar de las características negativas para la persona y su entorno (American Psychiatric Association, 2013) y, por otro a las dificultades ejecutivas que obstaculizan el monitoreo de sus estrategias y conductas.

En relación al proceso de almacenamiento, no se encontraron diferencias. Podría pensarse que, a pesar de los problemas en la organización y codificación, las personas con diagnóstico de trastorno por consumo de cocaína, no pierden la información que logran almacenar. En este punto, considerando las dificultades en el uso sistemático de estrategias semánticas y el sostenimiento de un recuerdo de tipo repetitivo, ligado a lo inmediato, resultaría válido preguntarse qué pasaría con ese recuerdo si se considerara un lapso de tiempo superior o si se aumentara la cantidad de información recibida. Puede hipotetizarse que el mismo no se sostendría en el tiempo y/o incrementaría el número de errores.

En relación al proceso de recuperación, la población clínica presenta un uso significativamente menor de estrategias semánticas en el recuerdo libre a corto plazo y en el recuerdo libre a largo plazo dando cuenta de problemas en el proceso de recuperación, pero en forma subsidiaria a las dificultades en el proceso de codificación. Estos resultados señalan dificultades en el funcionamiento estratégico u organizativo del procesador central, vinculado al uso de estrategias de almacenamiento y recuperación (Benedet \& Alejandre, 1998). Esto es, no pueden valerse de claves semánticas para recordar la información presentada porque, en general, no la organizaron ni la almacenaron de esa manera. No puede recodarse una información por categorías si no se codificó y almacenó con esa estructura. Asimismo, las diferencias en el índice que vincula el desempeño en la prueba de reconocimiento frente al recuerdo con claves a largo plazo ( $A C / R C l-L P)$ suma evidencia a que el uso escaso de estrategias semánticas en el proceso de codificación condiciona que no puedan valerse de ellas como estrategias organizativas, no pudiendo mejorar su desempeño al ser presentadas externamente.

Los índices analizados, constituyen parte del conjunto de índices del TAVEC que valoran procesos de recuperación activa, altamente dependientes del uso efectivo de estrategias de organización de la información, vinculadas a procesos ejecutivos.

Por último, al analizar en forma conjunta los índices de aciertos en la lista de reconocimiento y sesgo, representativos de procesos de recuperación pasiva, las personas con diagnóstico de trastorno por consumo de cocaína presentan un buen desempeño, estableciendo que la información almacenada puede ser reconocida. Asimismo, los índices de recuerdo libre corto plazo y recuerdo libre largo plazo, que indican la cantidad de palabras recordadas de la lista A sin ayuda de claves semánticas, y el índice que vincula la capacidad de reconocimiento frente al 
desempeño en la prueba de recuerdo libre a largo plazo (AC/RL-LP), no evidencian resultados significativamente desfavorables para el grupo clínico. Podría pensarse que las estrategias de memorización y repetición utilizadas, resultan en cierta medida efectivas en el corto plazo, aunque podrían tornarse ineficientes en un lapso de tiempo mayor. Asimismo, al considerar la calidad de dicho recuerdo, nuevamente se observa un número superior de errores.

Lo expuesto podría señalar que el desempeño de las personas con diagnóstico de trastorno por consumo de cocaína se encuentra menos afectado en tareas simples, como las de reconocimiento, ya que se reducen las demandas sobre las funciones ejecutiva (Gershberg \& Shimamura, 1985). Sin embargo, su desempeño tienda a empeorar en tareas de mayores exigencias ejecutivas. Como fue mencionado, el consumo crónico de cocaína genera alteraciones en las vías dopaminérgicas, que desempeñan un papel fundamental en el correcto funcionamiento de las funciones cognitivas en general y de las funciones ejecutivas en particular. Las dificultades reportadas por esta población para inhibir, flexibilizar y monitorear (GonzálezRoscigno et. al, 2015), pueden comprometer los procesos de supervisión y control de la conducta requeridos para un correcto funcionamiento de la memoria (García-Fernández et al., 2010).

\section{Conclusiones}

En conclusión, los resultados encontrados parecen sugerir que las personas con diagnóstico de trastorno por consumo de cocaína presentan un déficit específico y primario en los procesos de codificación, que genera en forma secundaria dificultades en los procesos de recuperación activa. Como en la instancia de aprendizaje no organizan semánticamente la información, tampoco pueden utilizar de manera sistemática este criterio con objeto de recuperarla. Esto conlleva un recuerdo marcado por la memorización inmediata y la repetición, más que por el análisis, la comprensión y la organización, lo que genera un mayor número de errores. En este punto, cabe destacar que estas dificultades en los procesos de memoria, podrían ser subsidiarias de fallas atencionales y ejecutivas reportadas en esta población (González-Roscigno et al., 2015; Romero-Ayuso et al., 2016).

Si bien los resultados encontrados constituyen hallazgos parciales, que deben continuar profundizándose, adquieren relevancia desde la lógica del modelo multinivel. Desde esta perspectiva es fundamental considerar las alteraciones cerebrales que el consumo de cocaína produce, los déficits neuropsicológicos que trae aparejados, y cómo ambas cuestiones repercuten en el comportamiento de las personas con consumo problemático, afectando directamente su estatus de salud y la evolución de los tratamientos. Las personas consumidoras de cocaína presentan alteraciones significativas y estables en diversas regiones del córtex prefrontal, que conjuntamente con estructuras del lóbulo medial temporal son fundamentales para el funcionamiento de la memoria (Kim \& Yoon, 1998, Verdejo et al., 2007). La ME, como proceso cognitivo, permite que una persona recuerde e incluso re-experimente sucesos del pasado y, es a su vez es base para otros procesos cognitivos como el lenguaje o el razonamiento. Constituye en sí misma una función vital para la supervivencia y la adaptación de una persona a su entorno (Ballesteros-Giménez, 2012), ya que posibilita organizar la información, de modo que la misma esté disponible para su utilización en situaciones futuras.

En relación a lo expuesto, resulta fundamental en el curso de un tratamiento para el consumo problemático, fomentar y estimular procesos más conscientes, reflexivos y controlados. Esto favorece la capacidad de organización de la información, el establecimiento de objetivos y metas, la inhibición de los estímulos que implican satisfacciones inmediatas, así como el 
monitoreo de la propia conducta y sus consecuencias para el desarrollo proyectos de vida saludables en el largo plazo. Asimismo, resulta beneficiosos el seguimiento de rutinas y estructuras que posibiliten un funcionamiento diario ordenado, productivo y satisfactorio.

Las conclusiones presentadas no deben considerarse definitivas ni libres de objeciones y por lo tanto no son fácilmente generalizables a otras poblaciones. En primer lugar, resulta necesario aumentar el tamaño muestral alcanzando un número de participantes que permita conformar grupos diferenciales y comparables en variables como edad, tiempo de consumo, tipo de sustancia consumida, entre otras. Por otra parte, resultaría interesante analizar el tiempo de permanencia en el tratamiento, realizando evaluaciones longitudinales en diferentes momentos del mismo, hecho que permitiría analizar si observa mejoría en el desempeño del funcionamiento de la ME en los tiempos de no consumo. Asimismo, en estudios futuros se podría incluir medidas de procesos atencionales y ejecutivos.

\section{Referencias}

American Psychiatric Association (APA). (2013). Manual diagnóstico y estadístico de los trastornos mentales, quinta edición, DSM-5. Barcelona: Masson.

Abeldaño, R. A., Fernández, A. R., Ventura, C. A. \& Estario, J. C. (2013). Consumo de sustancias psicoactivas en dos regiones argentinas y su relación con indicadores de pobreza. Cadernos de Saúde Pública, 29(5), 899-908. doi: 10.1590/\$0102-311X2013000500007

Ballesteros-Jiménez, S. (2012). Psicología de la memoria: Estructuras, procesos, sistemas. 1a ed. Madrid: Universidad Nacional de Educación a Distancia-Univérsitas.Benedet, M. J. \& Alejandre, M. A. (1998). Test de aprendizaje verbal España Computense (TAVEC). Madrid: TEA Ediciones. S. A.

Benedet, M. J., Martínez-Arias, R. \& Alejandre, M. A. (1998). Diferencias con la edad en el uso de estrategias, en el aprendizaje y en la retención. Anales de Psicología, 14(2), 139-156. Recuperado de https://revistas.um.es/analesps/article/view/31351

Bonet-Álvarez, J, Salvador-Castellano, A., Torres-Rivas, C., Aluco-Sánchez, E., Cano-Vega, M. \& Palma-Sevillano, C. (2015). Consumo de cocaína y estado de las funciones ejecutivas. Revista Española de Drogodependencias, 40(2), 13-23. Recuperado de https://www.aesed.com/upload/files/vol-40/n-2/v40n2 1.pdf

Bugge, M., Fontana, F., Ortiz de Latierro, S., Sarcona, E., Wydler, C., Aguilera, V.... Viñas, A. (2017). Prevalencia del uso de sustancias específicas potencialmente nocivas en adultos de 18 a 60 años. Revista Argentina de Medicina, 5(3), 159-167. Recuperado de http://www.revistasam.com.ar/revistasam.com.ar/index.php/RAM/article/view/145

Collette, F., Hogge, M., Salmon, E. \& Van Der Linden, M. (2006). Exploration of the neural substrates of executive functioning by functional neuroimaging. Neuroscience, 139(1), 209221. doi:10.1016/i.neuroscience.2005.05.035

Cunha, P. J, Nicastri, S., Gomes, L. P., Moino, R. M. \& Peluso, M. A. (2004) Neuropsychological impairments in crack cocaine dependent inpatients: preliminary findings. Revista Brasileira de Psiquiatría, 26(2), 103-106. doi: 10.1590/s1516-44462004000200007 
Delis, D. C., Kramer, J. H., Kaplan, E. \& Ober, B. A (1987). California Verbal Learning Test. San Antonio: The Psychological Corporation.

Einstein, G. O. \& Hunt, R. (1980). Levels of processing and organization: Additive effects of individual-item and relational processing. Journal of Experimental Psychology: Human Learning and Memory, 6(5), 588-598. doi: 10.1037/0278-7393.6.5.588

Fajardo-Balbuca, M. F., Carmilema-Tapia, J. C. \& Sacoto-Molina, A. M. (2018). Evaluación de atención y memoria en sujetos drogodependientes. Revista de la Facultad de Ciencias Médicas de la Universidad de Cuenca, 36(2), 37-45. Recuperado de https://publicaciones.ucuenca.edu.ec/ojs/index.php/medicina/article/view/2493

Fernández-Artamendi, S. \& Weidberg, S. (2016). Avances en la evaluación de las adicciones. Papeles del Psicólogo, 37(1), 52-61. Recuperado de http://www.redalyc.org/pdf/778/77844204007.pdf

Frith, C. \& Frith, U. (2010). Learning from Others: Introduction to the Special Review Series on Social Neuroscience. Neuron 65, 739-743. doi:10.1016/j.neuron.2010.03.015.

García-Fernández, G., García-Rodríguez, O. \& Secades-Villa, R. (2011). Neuropsicología y adicción a drogas. Papeles del Psicólogo, 32(2), 159-165. Recuperado de https://www.redalyc.org/pdf/778/77818544005.pdf

González-Roscigno, E. A., Mujica-Díaz, A. L., Terán-Mendoza, O. E., Guerrero-Alcedo, J. M. \& Arroyo-Alvarado, D. J. (2016). Sintomatología frontal y trastornos de personalidad en usuarios de drogas ilícitas. Drugs and Addictive Behavior, 1(1), 17-28. Doi: $\underline{10.21501 / 24631779.1755}$

Gershberg, F. B. \& Shimamura, A. P. (1995). Impaired use of organizational strategies in free recall following frontal lobe damage. Neuropsychologia, 33(10), 1305-1333. doi: /10.1016/00283932(95)00103-A

Hernández-Sampieri, R., Fernández-Collado, C. \& Baptista-Lucio, P. (2014). Metodología de la Investigación. México: Mc Graw Hill.

Introzzi, I. M., Urquijo, S., Richard's, M. M., Canet-Juric, L. \& Richaud, M. C. (2012). Función ejecutiva y uso de estrategias semánticas en niños. Revista Latinoamericana de Psicología, 44(3), 31-40.

Kim, J. J. \& Yoon, K. S. (1998). Stress: metaplastic effects in the hippocampus. Trends in neurosciences, 21(12), 505-509. doi: 10.1016/s0166-2236(98)01322-8

Kübler, A., Murphy, K., \& Garavan, H. (2005). Cocaine dependence and attention switching within and between verbal and visuospatial working memory. European Journal of Neuroscience, 21, 1984-1992. doi:10.1111/j.1460-9568.2005.04027.x

López, G. F. \& Muños, M. Y. (2012). Aprender a aprender; algunas aproximaciones practicas al fenómeno del aprendizaje. Revista Universidad Eafit, 95, 17-25. 
Lorea, I., Fernández-Montalvo, J., Tirapú-Ustarroz, J., Landa, N. \& López-Goñi, J. J. (2010). Rendimiento neuropsicológico en la adicción a la cocaína: una revisión crítica. Revista Neurología, 51, 412-426. Recuperado de https://www.researchgate.net/profile/Inaki Lorea/publication/297281148 Neuropsychol ogical performance in cocaine addiction a critical review/links/570b8a6808ae2eb942 239280/Neuropsychological-performance-in-cocaine-addiction-a-critical-review.pdf

Macbeth, G., Razumiejczyk, E. \& Ledesma, R. (2011). Cliff's Delta Calculator: A non-parametric effect size program for two groups of observations. Universitas Psychologica, 10(2), 545555. doi: 10.11144/javeriana.upsy10-2.cdcp

Madoz-Gúrpide, A., Ochoa-Mangado, E. \& Martínez-Pelegrini, B. (2009). Consumo de cocaína y daño neuropsicológico. Implicaciones clínicas. Medicina Clínica, 132(14), 555-559. doi: 10.1016/i.medcli.2008.07.019

Madoz-Gúrpide, A. \& Ochoa-Mangado, E. (2012). Alteraciones de funciones cognitivas y ejecutivas en pacientes dependientes de cocaína: estudio de casos y controles. Revista Neurología, 54(4), 199-208. Recuperado de https://socidrogalcohol.org/wpcontent/Pdf/publicaciones/cocaina/documentos/alteraciones-cognitivas-cocaina2012.pdf

Mendoza-Carmona, Y. L., López-Guerra, V. M. \& Cuello-Prato, P. V. (2019). Sobre la sintomatología prefrontal y las adicciones en la vida cotidiana. Revista Electrónica de Psicología Iztacala 22(1), 187-205. Recuperado de https://www.iztacala.unam.mx/carreras/psicologia/psiclin/vol22num1/Vol22No1Art11.pd f

Montgomery, C., Fisk, J. E., Newcombe, R., \& Murphy, P. N. (2005). The differential effects of ecstasy/polydrug use on executive. Psychopharmacology, 182, 262- 276. doi:10.1007/s00213-005-0065-9

Montoya-Filardi, A. \& Mazón, M. (2016). El cerebro adicto: imagen de las complicaciones neurológicas por el consumo de droga. Radiología, 1-14. doi: 10.1016/j.rx.2016.09.005

Organización Mundial de la Salud (2008). The global burden of disease: 2004 update. Switzerland: Organización Mundial de la Salud.

Pardo, A. \& Ruiz, M. (2002). Spss 11 guía para el análisis de datos. Análisis no paramétrico. México, D. F.: Mc Graw Hill.

Romano, J., Kromrey, J. D., Coraggio, J., Skowronek, J. \& Devine, L. (2006, octubre). Exploring methods for evaluating group differences on the NSSE and other surveys: Are the t-test and Cohen's $d$ indices the most appropriate choices? En Annual Meeting of the Southern Association for Institutional Research, Arlington, Virginia. Recuperado de http://citeseerx.ist.psu.edu/viewdoc/download?doi=10.1.1.595.6157\&rep=rep1\&type=p $\underline{\mathrm{df}}$

Romero-Ayuso, D., Mayoral-Gontán, Y. \& Triviño-Juárez, J. M. (2016) Inteligencia emocional y percepción de riesgo en consumidores de cocaína. Actas Españolas de Psiquiatría, 44(2), 
72-78. Recuperado de https://www.actaspsiquiatria.es/repositorio/18/100/ESP/18-100ESP-72-8-699330.pdf

Secretaria de Políticas Integrales sobre Drogas de la Nación Argentina (SEDRONAR, 2017a). Estudio Nacional en población de 12 a 65 años sobre consumo de sustancias psicoactivas. Argentina, 2017. Factores de riesgo en el consumo de sustancias psicoactivas. Recuperado de

http://www.observatorio.gov.ar/media/k2/attachments/FactoresZdeZRiesgoZZ2017ZZ3Z enero.pdf

Secretaria de Políticas Integrales sobre Drogas de la Nación Argentina (SEDRONAR, 2017c) Ventanas epidemiológicas en Dispositivo Integral de Abordaje Territorial. Recuperado de http://www.observatorio.gov.ar/media/k2/attachments/DIATZmarZdelZplata.pdf

Serper, M.R. Bergman, A., Copersino, M. L. James C.Y., Chou, J. C. Y,... Cancro, R. (2000). Learning and memory impairment in cocaine-dependent and comorbid schizophrenic patients. Psychiatry Research, 93(1), 21-32 doi: 10.1016/S0165-1781(99)00122-5.

Sohlberg, M. M. \& Mateer, C. A. (2001). Cognitive rehabilitation: an integrative neuropsychological approach. New York: Guilford Press.

Spronk, D. B., van Wel, J. H. P., Ramaekers, J. G. \& Verkes, R. J. (2013). Characterizing the cognitive effects of cocaine: A comprehensive review. Neuroscience and Biobehavioral Reviews, 37, 1838-1859. doi: 10.1016/i.neubiorev.2013.07.003

Tulving, E. (2002). Episodic Memory: From mind to brain. Annual Review of Psychology, 53, 1-25. doi: 10.1146/annurev.psych.53.100901.135114

Tulving, E. (2005). Episodic memory and autonoesis: Uniquely Human? En H. Terrace y J. Metcalfe (Eds.). The missing link in cognition (pp.3-56). Oxford: Oxford University Press.

Vallejo-Reyes, F. (2019). Evaluación de la Función Ejecutiva en usuarios con dependencia de pasta base de cocaína mediante una batería neuropsicológica. Psykhe, 28(1), 1-17. doi: 10.7764/psykhe.28.1.1111

Verdejo-García, A., Pérez-García, M., Sánchez-Barrera, M., Rodríguez-Fernández, A. \& Gómez-Río, M. (2007). Neuroimagen y drogodependencias: correlatos neuroanatómicos del consumo de cocaína, opiáceos, cannabis y éxtasis. Revista Neurología, 44(7), 432-439. Recuperado de http://www.neurologia.com/pdf/Web/4407/x070432.pdf

Vergara-Moragues, E., González-Saiz, F., Lozano-Rojas, O., Bilbao-Acedos, I., Fernández-Calderón, F., Betanzos-Espinosa, P., ... Pérez-García, M. (2010). Diagnóstico del trastorno por déficit de atención e hiperactividad (TDAH) en pacientes adultos con dependencia de cocaína: utilidad de los nuevos síntomas de funcionamiento ejecutivo de Barkley. Trastornos adictivos, 12(2), 72-78. doi: 10.1016/S1575-0973(10)70014-3

Vonmoos M, Hulka L. M, Preller K. H., Minder F., Baumgartner, M. R., \& Quednow, B. B. (2014). Cognitive Impairment in Cocaine Users is Drug-Induced but Partially Reversible: Evidence from a Longitudinal Study. Neuropsychopharmacology, 39(9), 2200-10. 